\title{
Numerical Solutions of MHD Viscous Flow of Newtonian Fluids due to a Shrinking Sheet by SOR Iterative Procedure
}

\author{
Mohammad Shafique ${ }^{1, *}$, Fatima Abbas ${ }^{2}$ \\ ${ }^{1}$ Ex-AP, Department of Mathematics, Gomal University, D I Khan, Pakistan \\ ${ }^{2}$ Department of Mathematics, Gomal University, D I Khan, Pakistan
}

Copyright $(0) 2015$ Horizon Research Publishing All rights reserved.

\begin{abstract}
The problem of Magneto Hydrodynamic viscous flow due to a shrinking sheet of Newtonian fluids has been solved numerically by using SOR Iterative Procedure. The similarity transformations have been used to reduce the highly nonlinear partial differential equations of motion to ordinary differential equations. The results have been calculated on three different grid sizes to check the accuracy of the results. The numerical results for Newtonian fluids are found in good agreement with those obtained by the previous results.
\end{abstract}

Keywords Newtonian Fluids, Shrinking Sheet and SOR Iterative Procedure

AMS Subject Classification: 76D99. 76M20, 65N22

\section{Introduction}

The flow problems of stretching surfaces have relevance to several technological processes. Chiam [1] investigated steady two dimensional stagnation point flow of an incompressible fluid towards a stretching surface. Mahapatra and Gupta $[2,3]$ combined both the stagnation point flow and stretching surface. Shafique and Rashid [4] examined the three dimensional fluid motion caused by the stretching of a flat surface. In recent years, the flow problem is being investigated for shrinking boundaries. Wang [5] studied the stagnation flow towards a shrinking sheet. He considered the two dimensional and axisymmetric studies of this problem. His results represent a rare class of exact similarity solutions with reverse flow. Fang and Zhang [6] considered MHD flow over a shrinking sheet and obtained closed form exact solution for the problem. The MHD boundary layer flow of fluid over a shrinking sheet has been studied by Hayat et al [7] and Fang [8]. Nadeem et al [9] and Ara et al [10] have been investigated MHD boundary layer flow of fluid over an exponentially permeable shrinking sheet. The steady boundary layer flow and steady two-dimensional flow of a nanofluid past a nonlinearly permeable stretching/ shrinking sheet is numerically studied by Zaimi et al $[11,12]$. Sajid and Hayat [13] applied homotopy analysis method for MHD viscous flow due to a shrinking sheet. The problem of [13] is studied by Noor et al. [14] by using simple non-perturbative method.

In this research, the numerical solutions of MHD viscous flow due to a shrinking sheet for Newtonian fluid have been discussed. In order to find the numerical solution of the problem, the Navier Stokes equations are reduced to ordinary differential equations by using similarity transformations [13]. This system is solved numerically by using SOR Iterative Procedure with Simpson (1/3) Rule. The calculations have been carried out using three different grid sizes to check the accuracy of the results. The present numerical results have also been compared with the previous results in a particular case and found in good agreement. The numerical results have been discussed in both tabular as well as graphically.

\section{Mathematical Analysis}

The continuity equation and the Navier-Stokes equations for incompressible fluid in the presence of body forces are given by

$$
\begin{gathered}
\frac{\partial \rho}{\partial t}+\nabla \cdot(\rho \underline{\mathbf{v}})=0, \\
\rho\left(\frac{\partial \underline{V}}{\partial t}+(\underline{V} \cdot \nabla) \underline{V}\right)=\underline{f}-\nabla p+\mu \nabla^{2} \underline{V}
\end{gathered}
$$

Where $\rho$ and $\mathbf{v}$ are respectively, the density and the velocity vector of the fluid.

The assumptions are made for the problem under consideration. The fluid flow is steady, laminar and incompressible. The fluid is electrically conducting in the presence of a magnetic field of strength $B_{0}$. The 
electromagnetic body force is given as $\underline{f}=-\sigma B_{0}^{2}(u, v, 0)$. The fluid flow in the frame of three dimensional Cartesian coordinate systems and

$\underline{\mathbf{V}}=(u(x, y, z), v(x, y, z), w(x, y, z))$ is the velocity field of flow.

Under the above assumptions, the equations (1) and (2) become

$$
\begin{gathered}
\frac{\partial u}{\partial x}+\frac{\partial v}{\partial y}+\frac{\partial w}{\partial z}=0 \\
u \frac{\partial u}{\partial x}+v \frac{\partial u}{\partial y}+w \frac{\partial u}{\partial z}=-\frac{1}{\rho} \frac{\partial p}{\partial x}+v\left(\frac{\partial^{2} u}{\partial x^{2}}+\frac{\partial^{2} u}{\partial y^{2}}+\frac{\partial^{2} u}{\partial z^{2}}\right)-\frac{\sigma B_{0}^{2}}{\rho} u \\
u \frac{\partial v}{\partial x}+v \frac{\partial v}{\partial y}+w \frac{\partial v}{\partial z}=-\frac{1}{\rho} \frac{\partial p}{\partial y}+v\left(\frac{\partial^{2} v}{\partial x^{2}}+\frac{\partial^{2} v}{\partial y^{2}}+\frac{\partial^{2} v}{\partial z^{2}}\right)-\frac{\sigma B_{0}^{2}}{\rho} v \\
u \frac{\partial w}{\partial x}+v \frac{\partial w}{\partial y}+w \frac{\partial w}{\partial z}=-\frac{1}{\rho} \frac{\partial p}{\partial z}+v\left(\frac{\partial^{2} w}{\partial x^{2}}+\frac{\partial^{2} w}{\partial y^{2}}+\frac{\partial^{2} w}{\partial z^{2}}\right)
\end{gathered}
$$

Where $p$ is the pressure and $v=\frac{\mu}{\rho}$ is Kinematics Viscosity and $\mu$ denotes viscosity coefficient. The boundary conditions are

$$
\left.\begin{array}{l}
\begin{array}{l}
u=a x, v=-a(m-1) y, w=-W \\
u \rightarrow 0 \text { as } y \rightarrow \infty
\end{array} \text { at } y=0,
\end{array}\right\}
$$

Here $a>0$ is the shrinking constant and $W$ is the suction velocity. When $m=1$, the sheet shrinks in the x-direction and when $m=2$, the sheet shrinks axisymmetrically.

In order to solve equations (3) to (6), the similarity transformations of [13] are given:

$$
u=x a f^{\prime}(\eta), v=y(m-1) a f^{\prime}(\eta), w=-m \sqrt{\mathrm{a} v} f(\eta),
$$

where $\eta=\sqrt{\frac{a}{v}} z$ is a dimensionless variable. The continuity equation (3) satisfied and the equation (6) can be integrated to give $\frac{p}{\rho}=v \frac{\partial w}{\partial z}-\frac{w^{2}}{2}+$ constant .

The resulting partial differential equations by using (8) we obtain:

$$
f^{\prime \prime \prime}-M^{2} f^{\prime}-f^{2}+m f f^{\prime \prime}=0,
$$

with the boundary conditions:

$$
\left.\begin{array}{l}
f=S, f^{\prime}=-1 \text { at } \eta=0, \\
f^{\prime} \rightarrow 0 \text { as } \eta \rightarrow \infty .
\end{array}\right\}
$$

Where ${ }_{s=\frac{W}{m \sqrt{a v}}}$ and $\mathrm{M}^{2}=\frac{\sigma B_{0}^{2}}{\rho a}$. The prime denotes the differentiation with respect to $\eta$.

\section{Finite Difference Equations}

For numerical purpose, we rewrite the equations (9) and (10) by putting

$$
P=f^{\prime}
$$

The equation (9) as follows:

$$
P^{\prime \prime}-M^{2} P-P^{2}+m f P^{\prime}=0
$$

and the boundary conditions (10) takes the form:

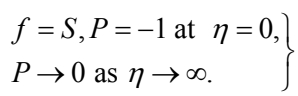

Now if we approximate equation (12) by central difference approximations at a typical point $\eta=\eta_{n}$ of the interval $[0, \infty)$, we obtained:

$$
\begin{aligned}
& \frac{P_{n+1}-2 P_{n}+P_{n-1}}{h^{2}}-M^{2} P_{n}+m f_{n}\left\{\frac{P_{n+1}-P_{n-1}}{2 h}\right\}-P_{n}^{2}=0, \\
& \Rightarrow\left(4+2 h^{2} M^{2}+2 h^{2} P_{n}\right) P_{n}=2\left(P_{n+1}+P_{n-1}\right)+m h f_{n}\left(P_{n+1}-P_{n-1}\right) .
\end{aligned}
$$

Where $h$ denotes a grid size and the symbols used denote as $f_{n}=f\left(\eta_{n}\right), P_{n}=P\left(\eta_{n}\right)$, at a typical point of the interval $[0, \infty)$ replaced by $[0, \beta)$, where $\beta$ is sufficiently large.

\section{Computational Procedure}

We now integrate numerically equation (11) and solve the system of finite difference equation (14) at each required grid point of the interval $[0, \infty)$. The equation (11) is integrated by using the Simpson's (1/3) rule [15] as given below at the gird point $\eta=\eta_{n}$

$$
f_{n+1}=f_{n-1}+\frac{h}{3}\left\{P_{n-1}+4 P_{n}+P_{n+1}\right\} \text {. }
$$

Whereas the system of finite-difference equations (14) are solved by using S.O.R. iterative procedure [15]

$$
P_{n}=\frac{\omega}{\left(4+2 h^{2} M^{2}+2 h^{2} P_{n}\right)}\left\{2\left(P_{n+1}+P_{n-1}\right)+m h f_{n}\left(P_{n+1}-P_{n-1}\right)\right\}+(1-\omega) P_{n} .
$$

Subject to the appropriate boundary conditions (13), the computation has been checked for different of the relaxation parameter $\omega$ between 1 and 2. The optimum value of the relaxation parameter for the problem under consideration is 1.5 .

The SOR iterative procedure is terminated when the following criterion is satisfied:

$$
\max _{i=1}^{n}\left|U_{i}^{n+1}-U_{i}^{n}\right|<10^{-6}
$$

where $n$ denotes the number of iterations and $U$ stands for each of $P$ and $f$. The stability of SOR iterative procedure and accuracy of the solution has been checked for step sizes $h=0.01,0.005$ and 0.0025 and it is not necessary to discuss because this study has been focus over numerical solution of the problem however accuracy is excellent. 


\section{Numerical Results and Discussions}

The numerical computation has been performed to study the effect of the flow parameters namely $\mathrm{S}$ and $\mathrm{M}$. The accuracy of the results is checked by comparing them on different grid sizes. The results for the non-dimensional velocity components $f, f^{\prime}$ are shown in the tables 1-3, for each of grid sizes mentioned above. When $m=1$, the sheet shrinks in the $\mathrm{x}$-direction and when $m=2$, the sheet shrinks axisymmetrically. The results compare very well. The comparison of the present results for $f^{\prime \prime}(0)$ (the skin friction) with the previous results by $[13,14]$ is given in table 4 .

Graphically, the curves for the function $f, f^{\prime}$ have been plotted and are displayed in figures 1 to 8 for several values of the parameters $m, \mathrm{~S}$ and $\mathrm{M}$. When $m=1$, the two dimensional case, figures.1-2, with $\mathrm{M}=2$ and for different values of $S$, and figures $3-4$, with $S=1$ and for different values of $\mathrm{S}$. When $m=2$, the axisymmetric case, figures.5-6, with $M=2$ and for different values of $S$, and figures 7-8, with $\mathrm{S}=1$ and for different values of $\mathrm{S}$.

It can be observed that the function $f^{\prime}$ increases with the increasing values of $\mathrm{S}$ at a fixed value of $\mathrm{M}$. The function $f$ decreases initially and then becomes uniform for all values of $\mathrm{S}$ and $\mathrm{M}$.

Consequently, the effects of different parameters are observed on the similarity $f$ and the velocity $f^{\prime}$. From this analysis, the boundary layer becomes thinner for larger $\mathrm{S}$. The increasing values of $M$ show stronger effect on both the velocity functions. Also, the present results for $f^{\prime \prime}(0)$ (the skin friction) have good agreement with the previous results.

Table 1. Numerical Results of $f$ and $f$ 'using SOR Iterative Procedure Simpson's Rule for specified vales of parameters $M=1.0$ and $\mathrm{S}=0.1,2.0$

\begin{tabular}{|c|c|c|c|c|c|c|c|c|c|}
\hline \multirow{3}{*}{$h$} & \multirow{3}{*}{$\eta$} & \multicolumn{4}{|c|}{$\mathrm{M}=1.0$ and $\mathrm{S}=0.1$} & \multicolumn{4}{|c|}{$\mathrm{M}=1.0$ and $\mathrm{S}=2.0$} \\
\hline & & \multicolumn{2}{|c|}{$m=1$} & \multicolumn{2}{|c|}{$m=2$} & \multicolumn{2}{|c|}{$m=1$} & \multicolumn{2}{|c|}{$m=2$} \\
\hline & & $f(\eta)$ & $f^{\prime}(\eta)$ & $f(\eta)$ & $f^{\prime}(\eta)$ & $f(\eta)$ & $f^{\prime}(\eta)$ & $f(\eta)$ & $f^{\prime}(\eta)$ \\
\hline \multirow{6}{*}{0.01} & 0.0 & 0.100000 & -1.000000 & 0.100000 & -1.000000 & 2.000000 & -1.000000 & 2.000000 & -1.000000 \\
\hline & 1.0 & -0.827905 & -0.858721 & -0.899999 & -0.999999 & 1.567687 & -0.135310 & 1.743410 & -0.022928 \\
\hline & 2.0 & -1.623252 & -0.734774 & -1.899999 & -0.999997 & 1.509199 & -0.018297 & 1.737431 & -0.000543 \\
\hline & 3.0 & -2.302482 & -0.625666 & -2.899992 & -0.999989 & 1.501299 & -0.002460 & 1.737289 & -0.000013 \\
\hline & 4.0 & -2.874224 & -0.511521 & -3.899951 & -0.999780 & 1.500250 & -0.000307 & 1.737287 & 0.000000 \\
\hline & 5.0 & -3.228485 & 0.000000 & -4.791556 & 0.000000 & 1.500141 & 0.000000 & 1.737287 & 0.000000 \\
\hline \multirow{6}{*}{0.005} & 0.0 & 0.100000 & -1.000000 & 0.100000 & -1.000000 & 2.000000 & -1.000000 & 2.000000 & -1.000000 \\
\hline & 1.0 & -0.825926 & -0.854981 & -0.900002 & -1.000004 & 1.567721 & -0.135268 & 1.743399 & -0.022929 \\
\hline & 2.0 & -1.616261 & -0.728699 & -1.900015 & -1.000032 & 1.509264 & -0.018278 & 1.737421 & -0.000542 \\
\hline & 3.0 & -2.288724 & -0.618366 & -2.900073 & -1.000084 & 1.501375 & -0.002453 & 1.737280 & -0.000013 \\
\hline & 4.0 & -2.852905 & -0.503858 & -3.900168 & -0.999945 & 1.500330 & -0.000306 & 1.737278 & 0.000000 \\
\hline & 5.0 & -3.201166 & 0.000000 & -4.791895 & 0.000000 & 1.500222 & 0.000000 & 1.737278 & 0.000000 \\
\hline \multirow{5}{*}{0.0025} & 1.0 & -0.792169 & -0.789780 & -0.899995 & -0.999989 & 1.568048 & -0.134888 & 1.743796 & -0.022638 \\
\hline & 2.0 & -1.491203 & -0.614091 & -1.899984 & -0.999982 & 1.509866 & -0.018100 & 1.737946 & -0.000507 \\
\hline & 3.0 & -2.031150 & -0.470543 & -2.899959 & -0.999959 & 1.502079 & -0.002407 & 1.737817 & -0.000010 \\
\hline & 4.0 & -2.437919 & -0.341138 & -3.899901 & -0.999755 & 1.501057 & -0.000297 & 1.737817 & 0.000000 \\
\hline & 5.0 & -2.660019 & 0.000000 & -4.791506 & 0.000000 & 1.500951 & 0.000000 & 1.737817 & 0.000000 \\
\hline
\end{tabular}

Table 2. Numerical Results of $f$ and $f$ 'using SOR Iterative Procedure Simpson's Rule for specified vales of parameters $M=2.0$ and $S=0.5,1.0$

\begin{tabular}{|c|c|c|c|c|c|c|c|c|c|}
\hline \multirow{3}{*}{$h$} & \multirow{3}{*}{$\eta$} & \multicolumn{4}{|c|}{$\mathrm{M}=2.0$ and $\mathrm{S}=0.5$} & \multicolumn{4}{|c|}{$\mathrm{M}=2.0$ and $\mathrm{S}=1.0$} \\
\hline & & \multicolumn{2}{|c|}{$m=1$} & \multicolumn{2}{|c|}{$m=2$} & \multicolumn{2}{|c|}{$m=1$} & \multicolumn{2}{|c|}{$m=2$} \\
\hline & & $f(\eta)$ & $f^{\prime}(\eta)$ & $f(\eta)$ & $f^{\prime}(\eta)$ & $f(\eta)$ & $f^{\prime}(\eta)$ & $f(\eta)$ & $f^{\prime}(\eta)$ \\
\hline \multirow{6}{*}{0.01} & 0.0 & 0.500000 & -1.000000 & 0.500000 & -1.000000 & 1.000000 & -1.000000 & 1.000000 & -1.000000 \\
\hline & 1.0 & 0.067665 & -0.135336 & 0.086229 & -0.123025 & 0.609164 & -0.099976 & 0.667411 & -0.061125 \\
\hline & 2.0 & 0.009157 & -0.018312 & 0.033743 & -0.016080 & 0.570091 & -0.009993 & 0.646618 & -0.003907 \\
\hline & 3.0 & 0.001243 & -0.002476 & 0.026857 & -0.002117 & 0.566186 & -0.000998 & 0.645287 & -0.000250 \\
\hline & 4.0 & 0.000175 & -0.000328 & 0.025953 & -0.000274 & 0.565797 & -0.000098 & 0.645202 & -0.000016 \\
\hline & 5.0 & 0.000050 & 0.000000 & 0.025849 & 0.000000 & 0.565763 & 0.000000 & 0.645198 & 0.000000 \\
\hline \multirow{6}{*}{0.005} & 0.0 & 0.500000 & -1.000000 & 0.500000 & -1.000000 & 1.000000 & -1.000000 & 1.000000 & -1.000000 \\
\hline & 1.0 & 0.067688 & -0.135308 & 0.086255 & -0.122990 & 0.609181 & -0.099959 & 0.667415 & -0.061121 \\
\hline & 2.0 & 0.009202 & -0.018297 & 0.033797 & -0.016061 & 0.570119 & -0.009987 & 0.646623 & -0.003907 \\
\hline & 3.0 & 0.001297 & -0.002470 & 0.026924 & -0.002110 & 0.566218 & -0.000997 & 0.645292 & -0.000250 \\
\hline & 4.0 & 0.000232 & -0.000327 & 0.026022 & -0.000272 & 0.565829 & -0.000098 & 0.645207 & -0.000016 \\
\hline & 5.0 & 0.000108 & 0.000000 & 0.025920 & 0.000000 & 0.565795 & 0.000000 & 0.645202 & 0.000000 \\
\hline \multirow{5}{*}{0.0025} & 1.0 & 0.067868 & -0.135075 & 0.086437 & -0.122742 & 0.609474 & -0.099573 & 0.667677 & -0.060841 \\
\hline & 2.0 & 0.009573 & -0.018163 & 0.034175 & -0.015926 & 0.570719 & -0.009774 & 0.647067 & -0.003809 \\
\hline & 3.0 & 0.001764 & -0.002411 & 0.027397 & -0.002054 & 0.566952 & -0.000927 & 0.645787 & -0.000231 \\
\hline & 4.0 & 0.000734 & -0.000311 & 0.026528 & -0.000257 & 0.566600 & -0.000082 & 0.645710 & -0.000013 \\
\hline & 5.0 & 0.000616 & 0.000000 & 0.026431 & 0.000000 & 0.566572 & 0.000000 & 0.645707 & 0.000000 \\
\hline
\end{tabular}


Table 3. Numerical Results of $f$ and $f$ 'using SOR Iterative Procedure Simpson's Rule for specified vales of parameters $M=3.0$ and $S=1.0,1.5$

\begin{tabular}{|c|c|c|c|c|c|c|c|c|c|}
\hline \multirow{3}{*}{$h$} & \multirow{3}{*}{$\eta$} & \multicolumn{4}{|c|}{$\mathrm{M}=3.0$ and $\mathrm{S}=1.0$} & \multicolumn{4}{|c|}{$\mathrm{M}=3.0$ and $\mathrm{S}=1.5$} \\
\hline & & \multicolumn{2}{|c|}{$m=1$} & \multicolumn{2}{|c|}{$m=2$} & \multicolumn{2}{|c|}{$m=1$} & \multicolumn{2}{|c|}{$m=2$} \\
\hline & & $f(\eta)$ & $f^{\prime}(\eta)$ & $f(\eta)$ & $f^{\prime}(\eta)$ & $f(\eta)$ & $f^{\prime}(\eta)$ & $f(\eta)$ & $f^{\prime}(\eta)$ \\
\hline \multirow{6}{*}{0.01} & 0.0 & 1.000000 & -1.000000 & 1.000000 & -1.000000 & 1.500000 & -1.000000 & 1.500000 & -1.000000 \\
\hline & 1.0 & 0.713634 & -0.034311 & 0.747957 & -0.021106 & 1.234863 & -0.025320 & 1.284418 & -0.010417 \\
\hline & 2.0 & 0.703809 & -0.001177 & 0.742572 & -0.000456 & 1.228150 & -0.000641 & 1.282151 & -0.000111 \\
\hline & 3.0 & 0.703472 & -0.000040 & 0.742456 & -0.000010 & 1.227981 & -0.000016 & 1.282127 & -0.000001 \\
\hline & 4.0 & 0.703461 & -0.000001 & 0.742453 & 0.000000 & 1.227977 & 0.000000 & 1.282127 & 0.000000 \\
\hline & 5.0 & 0.703461 & 0.000000 & 0.742453 & 0.000000 & 1.227977 & 0.000000 & 1.282127 & 0.000000 \\
\hline \multirow{6}{*}{0.005} & 0.0 & 1.000000 & -1.000000 & 1.000000 & -1.000000 & 1.500000 & -1.000000 & 1.500000 & -1.000000 \\
\hline & 1.0 & 0.713651 & -0.034303 & 0.747971 & -0.021101 & 1.234878 & -0.025311 & 1.284423 & -0.010416 \\
\hline & 2.0 & 0.703829 & -0.001176 & 0.742587 & -0.000456 & 1.228169 & -0.000640 & 1.282156 & -0.000110 \\
\hline & 3.0 & 0.703493 & -0.000040 & 0.742471 & -0.000010 & 1.228000 & -0.000016 & 1.282133 & -0.000001 \\
\hline & 4.0 & 0.703481 & -0.000001 & 0.742469 & 0.000000 & 1.227997 & 0.000000 & 1.282133 & 0.000000 \\
\hline & 5.0 & 0.703481 & 0.000000 & 0.742469 & 0.000000 & 1.227997 & 0.000000 & 1.282133 & 0.000000 \\
\hline \multirow{6}{*}{0.0025} & 0.0 & 1.000000 & -1.000000 & 1.000000 & -1.000000 & 1.500000 & -1.000000 & 1.500000 & -1.000000 \\
\hline & 1.0 & 0.713712 & -0.034254 & 0.748130 & -0.020959 & 1.234959 & -0.025248 & 1.284461 & -0.010396 \\
\hline & 2.0 & 0.703916 & -0.001167 & 0.742818 & -0.000433 & 1.228282 & -0.000630 & 1.282201 & -0.000109 \\
\hline & 3.0 & 0.703583 & -0.000039 & 0.742710 & -0.000008 & 1.228116 & -0.000015 & 1.282179 & -0.000001 \\
\hline & 4.0 & 0.703573 & -0.000001 & 0.742709 & 0.000000 & 1.228114 & 0.000000 & 1.282179 & 0.000000 \\
\hline & 5.0 & 0.703573 & 0.000000 & 0.742709 & 0.000000 & 1.228114 & 0.000000 & 1.282179 & 0.000000 \\
\hline
\end{tabular}

Table 4. Comparison of the Numerical Value of $f^{\prime \prime}(0)$ for $\mathrm{M}=2.0$

\begin{tabular}{|c|c|c|c|c|}
\hline \multirow{2}{*}{$\mathrm{S}$} & \multicolumn{2}{|c|}{$m=1$} & \multicolumn{2}{c|}{$m=2$} \\
\cline { 2 - 5 } & Present Results & Previous Results & Present Results & Previous Results \\
\hline 0.1 & 1.779055 & - & 1.734042 & - \\
\hline 0.5 & 1.995825 & - & 2.189207 & - \\
\hline 1.0 & 2.297425 & $2.30277[13]$ & 2.881837 & $2.89160[13]$ \\
\hline 1.5 & 2.630043 & - & 3.674960 & - \\
\hline 2.0 & 2.989721 & - & 4.530692 & - \\
\hline
\end{tabular}

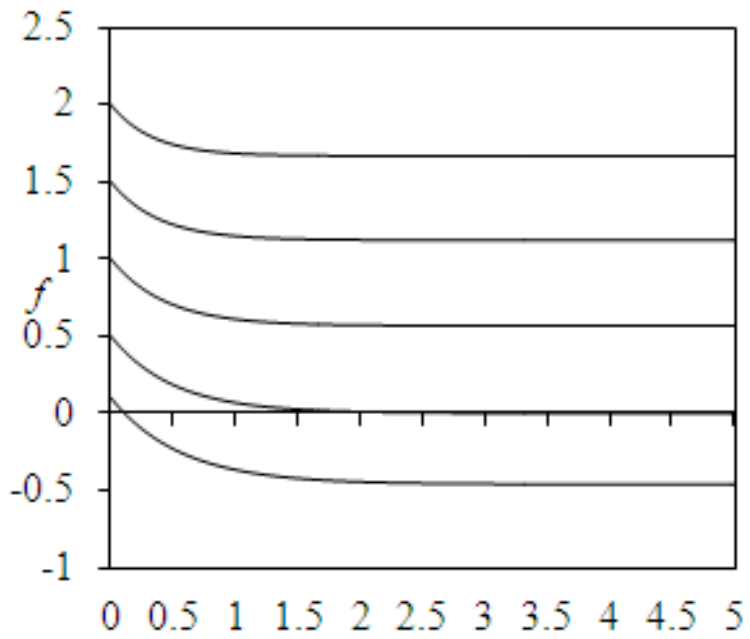

$\eta$

Figure 1. Graphs of the function $f(\eta)$ for $m=1, \mathrm{M}=2$ and $\mathrm{S}=.1, .5,1$, $1.5,2$. from bottom to top $\begin{array}{lllllllllll}0 & 0.5 & 1 & 1.5 & 2 & 2.5 & 3 & 3.5 & 4 & 4.5 & 5\end{array}$

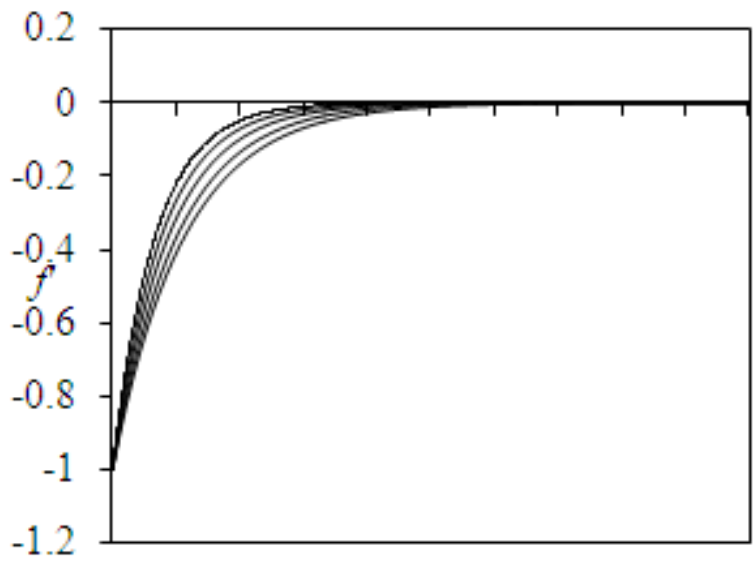

$\eta$

Figure 2. Graphs of the function $f^{\prime}(\eta)$ for $m=1, \mathrm{M}=2$ and $\mathrm{S}=.1, .5$, $1,1.5,2$. from bottom to top 


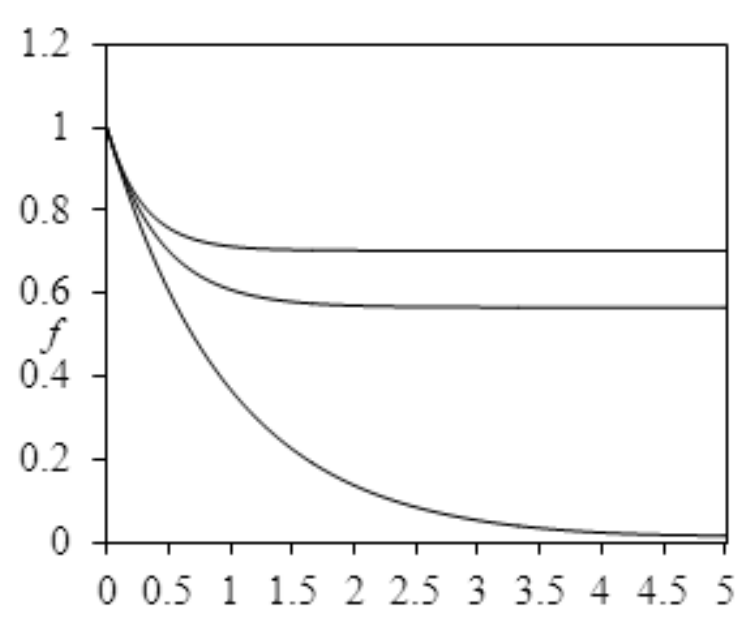

$\eta$

Figure 3. Graphs of the function $f(\eta)$ for $m=1, \mathrm{~S}=1$. and $\mathrm{M}=1,2,3$ from bottom to top

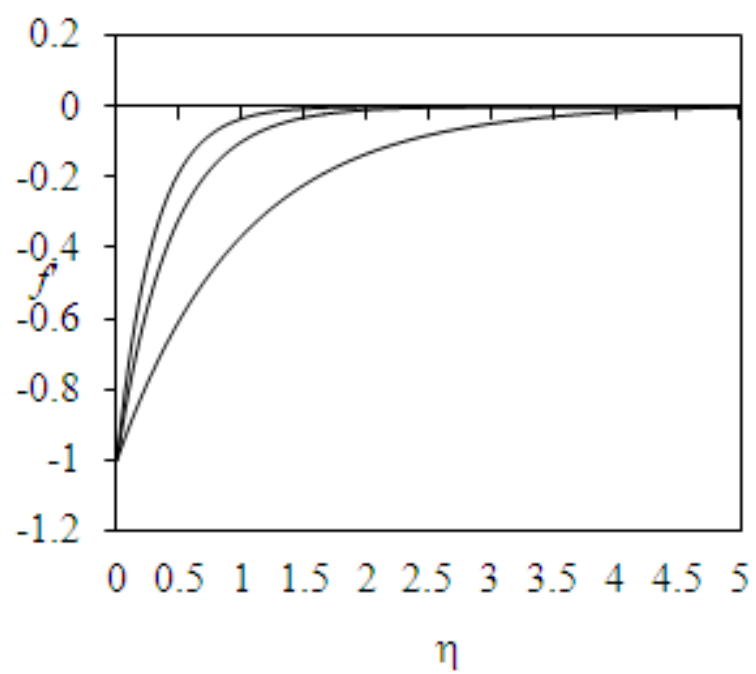

Figure 4. Graphs of the function $f^{\prime}(\eta)$ for $m=1, \mathrm{~S}=1$. and $\mathrm{M}=1,2,3$ from bottom to top

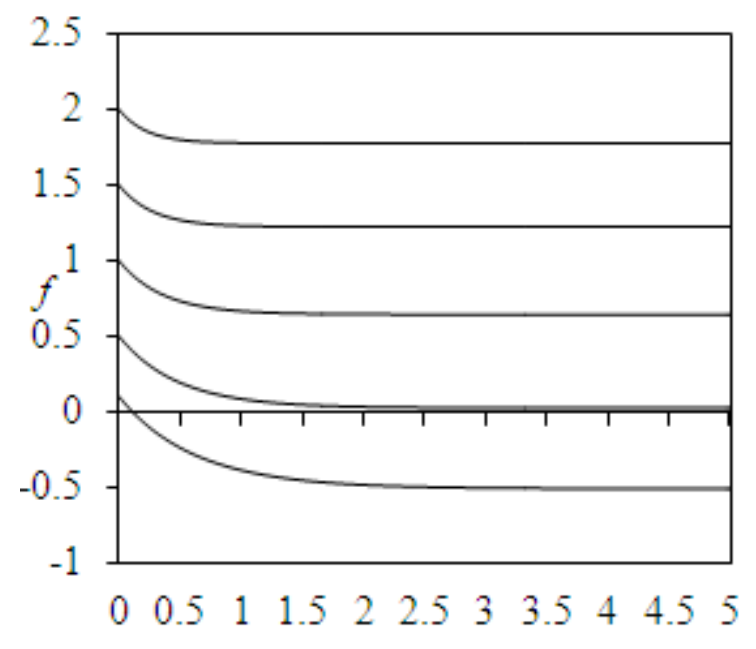

$\eta$

Figure 5. Graphs of the function $f(\eta)$ for $m=2, \mathrm{M}=2$ and $\mathrm{S}=.1, .5,1$, $1.5,2$. from bottom to top

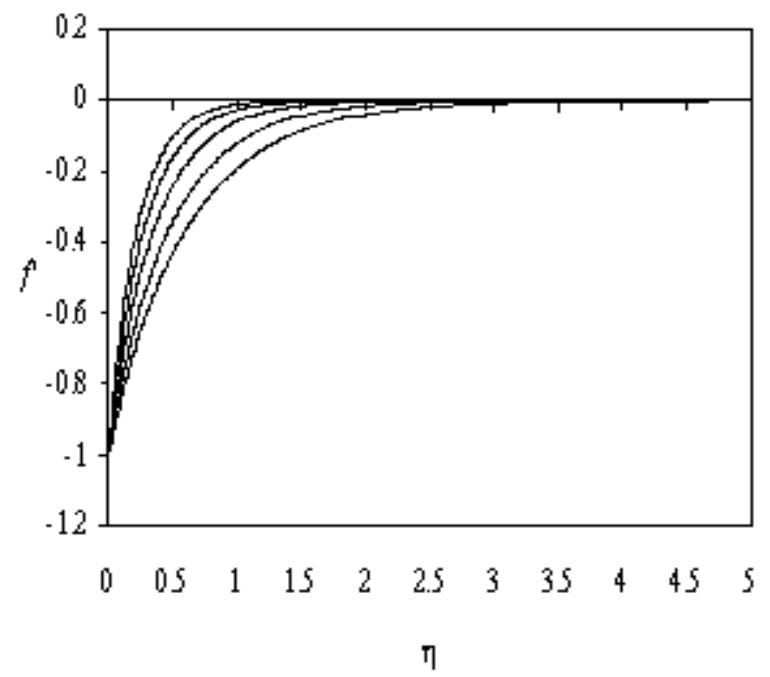

Figure 6. Graphs of the function $f^{\prime}(\eta)$ for $m=2, \mathrm{M}=2$ and $\mathrm{S}=.1, .5,1$, $1.5,2$. from bottom to top

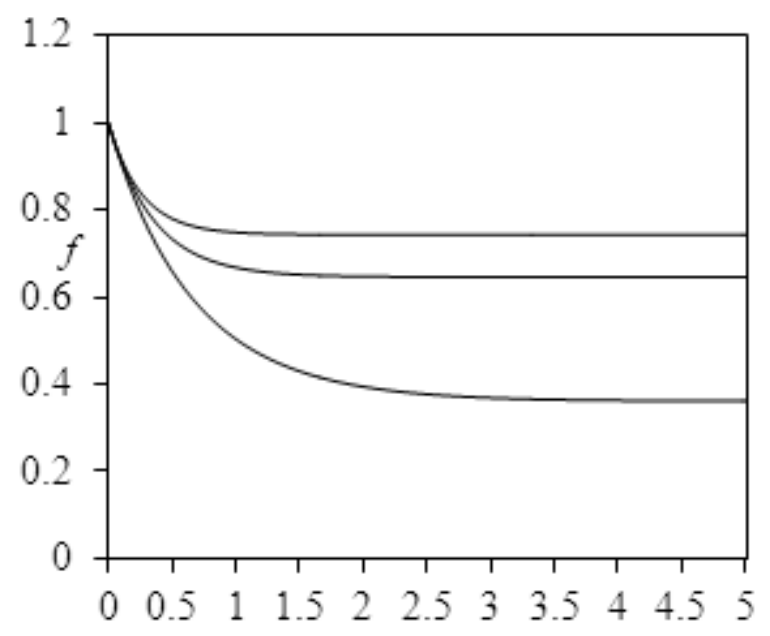

$\eta$

Figure 7. Graphs of the function $f(\eta)$ for $m=2, \mathrm{~S}=1$. and $\mathrm{M}=1,2,3$ from bottom to top

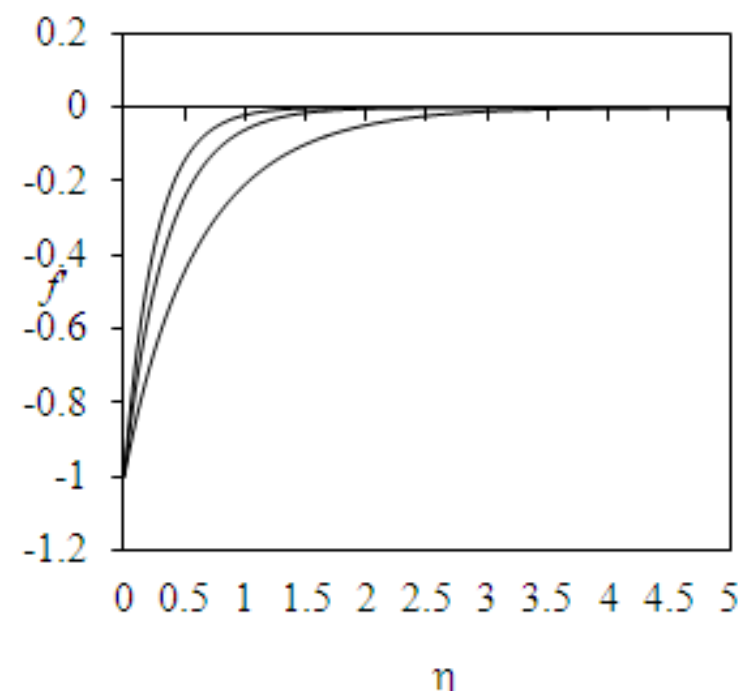

Figure 8. Graphs of the function $f^{\prime}(\eta)$ for $m=2, \mathrm{~S}=1$. and $\mathrm{M}=1,2,3$ from bottom to top. 


\section{REFERENCES}

[1] T. C. Chiam, Stagnation-Point flow towards a stretching plate, J. Phys. Soc. Japan 63 (1994) 2443-2444.

[2] T. R. Mahaputra and A. S. Gupta, Heat transfer in stagnation-point flow towards a stretching surface, Heat Mass Transfer, 38(2004)811-820.

[3] T. R. Mahaputra and A. S. Gupta, Stagnation-point flow towards a stretching surface, Can. J. Chem. Engg. 81(2003)258-263.

[4] M. Shafique and A. Rashid, The numerical methods for the 3dimensional flow due to a stretching flat surface Int. J. of Applied Math. 17(1) (2005) 15-26.

[5] Wang. C.Y. (2008), Stagnation flow towards a shrinking sheet, International journal of Non-Linear Mechanics, 43, 377 -382 .

[6] Fang, T. and Zhang, Ji. (2009), Closed- Form exact solutins of MHD viscous flow over a shrinking sheet, Commun Nonlinear Sci Numer Sumulat, 14, 2853 -2857.

[7] T. Hayat, Z. Abbas and M. Sajid, (2007), On the Analytic Solution of MHD Flow of a Second Grade Fluid Over a Shrinking Sheet, 1 .J. Appl. Mech. 74(6), 1165-1171

[8] Tiegang Fang, (2008), Boundary layer flow over a shrinking sheet with power-law velocity, International Journal of Heat and Mass Transfer, Volume 51, Issues 25-26, 5838-5843.

[9] S. Nadeem, Rizwan Ul Haq, C. Lee, (2012), MHD flow of a Casson fluid over an exponentially shrinking sheet,Scientia Iranica B 19 (6), 1550-1553.

[10] Asmat Ara, Najeeb Alam Khan, Hassam Khan, Faqiha Sultan, Radiation effect on boundary layer flow of an Eyring-Powell fluid over an exponentially shrinking sheet, (2014), Ain Shams Engineering Journal, Volume 5, Issue 4, 1337-1342.

[11] Khairy Zaimi, Anuar Ishak \& Ioan Pop, (2014), Boundary layer flow and heat transfer over a nonlinearly permeable stretching/shrinking sheet in a nanofluid, Scientific ReportsVolume:4, Article number:4404 DOI: doi:10.1038/ srep04404.

[12] Khairy Zaimi,, Anuar Ishak, Ioan Pop , (2014), Flow Past a Permeable Stretching/Shrinking Sheet in a Nanofluid Using Two-Phase Model, PLoS ONE 9(11): el11743.

[13] Sajid, M. and Hayat, T. (2009), The application of homotopy analysis method for MHD viscous flow due to a shrinking sheet, Chaors Soliton Fractals, 39, 1317-1323.

[14] Noor, N. F. M., Kechil, S. A. and Hashim, I. (2010), Simple non-perturbative solution for MHD viscous flow due to a shrinking sheet, Commun Nonlinear Sci Numer Sim Ilat 15, 144-148.

[15] C. F. Gerald, "Applied Numerical Analysis," AddisonWesley Publication, New York, 1989. 